\title{
Combination of interval set and soft set
}

\author{
Keyun Qin ${ }^{1 *}$ Dan Meng ${ }^{2}$, Zheng Pei ${ }^{3}$, Yang $\mathrm{Xu}^{1}$ \\ ${ }^{1}$ College of Mathematics, Southwest Jiaotong University, Chengdu, Sichuan, 610031, China, \\ E-mail: keyunqin@263.net(Qin),xuyang@home.swjtu.edu.cn(Xu) \\ ${ }^{2}$ School of Economics Information Engineering, Southwestern University of Finance and Economics, \\ Chengdu, Sichuan 611130,China, E-mail: danmeng2006@gmail.com \\ ${ }^{3}$ School of Mathematics \& Computer Engineering, Xihua University, Chengdu, Sichuan, 610039, China, \\ E-mail: pqyz@263.net
}

Received 1 January 2012

Accepted 19 November 2012

\begin{abstract}
Soft set theory and interval set theory are all mathematical tools for dealing with uncertainties. This paper is devoted to the discussion of soft interval set and its application. The notion of soft interval sets is introduced by combining soft set and interval set. Several operations on soft interval sets are presented in a manner parallel to that used in defining operations on soft sets and the lattice structures of soft interval sets are established. In addition, a soft interval set based decision making problem is analyzed.
\end{abstract}

Keywords: Soft set, Interval set, Soft interval set, Soft equality, Soft quotient algebra.

\section{Introduction}

To solve complicated problems in economics, engineering, environmental science and social science, methods in classical mathematics are not always successful because of various types of uncertainties present in these problems. While a wide range of theories such as probability theory, fuzzy set theory $^{1}$, rough set theory ${ }^{2}$, and other mathematical tools are well-known and often serve as useful approaches to describing uncertainty, each of them has its advantages as well as inherent difficulties. In 1999, Molodtsov ${ }^{3}$ introduced the concept of soft sets, which can be seen as a new mathematical tool for dealing with uncertainties. This so-called soft set theory seems to be free from the difficulties affecting the existing methods.

Presently, works on soft set theory are progress- ing rapidly. Soft set theory has a rich potential for applications in several directions, few of which had been shown by Molodtsov ${ }^{3}$. Maji et al. ${ }^{4}$ described the application of soft set theory to a decision making problem. Chen et al..$^{5}$ presented a new definition of soft set parameterization reduction, and compared this definition to the related concept of attributes reduction in rough set theory. Kong et al. ${ }^{6}$ introduced the notion of normal parameter reduction of soft sets and presented a heuristic algorithm to compute normal parameter reduction. In theoretical aspects, Maji et al. ${ }^{7}$ defined several operations on soft sets and made a theoretical study on the theory of soft sets. Irfan et al. ${ }^{8}$ introduced some new operations on soft sets and improved the notion of complement of soft set. Based on these operations, Qin et al. ${ }^{9}$ established the lattice structures and the soft quotient algebras of soft sets.

\footnotetext{
${ }^{*}$ Corresponding author.
} 
The study of hybrid models combining soft sets with other mathematical structures is emerging as an active research topic of soft set theory. Aktas and Cagman ${ }^{10}$ compared soft sets to the related concepts of fuzzy sets and rough sets. They also defined the notion of soft groups and derived some related properties. Jun ${ }^{11}$ introduced the notion of soft BCK/BCIalgebras. Jun and Park ${ }^{12}$ discussed the applications of soft sets in ideal theory of BCK/BCI-algebras. Maji et al. ${ }^{13}$ initiated the study on hybrid structures involving fuzzy sets and soft sets. They introduced the notion of fuzzy soft sets, which can be seen as a fuzzy generalization of soft sets. Roy et al. ${ }^{14}$ presented a novel approach to deal with fuzzy soft set based decision making problems. Yang et al. ${ }^{15}$ introduced the notion of interval-valued fuzzy soft sets by combining interval-valued fuzzy set and soft set models. By means of level soft sets and reduct fuzzy soft sets, Feng et al. ${ }^{16,17}$ presented some adjustable approaches to fuzzy soft set and intervalvalued fuzzy soft set based decision making problems. The combination of soft set and rough set models was also discussed by some researchers ${ }^{18,19}$.

The notion of interval sets was proposed by $\mathrm{Yao}^{20}$ as a mathematical tool for dealing with qualitative information. An interval set is represented by a pair of sets, namely, the lower and upper bounds. It provides a useful and convenient method for representing partially known concepts or for approximating undefinable concepts or complex concepts. A generalized decision logic in interval-set-valued information system is introduced ${ }^{21}$. Interval sets are closely related to, and complementary to, fuzzy sets and rough sets. The relationships among interval sets, rough sets and fuzzy sets were investigated ${ }^{22,23}$. A significant difference between these models lies in the definition and interpretation of their extended set-theoretic operators. More specifically, within a framework of possible-worlds semantics analysis, it has been shown that the rough set model and interval set model correspond to two different extended propositional logics 22 .

In many practical soft set based decision making problems, the parameter approximations are extremely individual (dependent on expert's evaluation of alternatives) and thus cannot be lightly confirmed.
Sometimes we can only provide a lower bound and an upper bound of parameter approximations. This leads to the motivation to combine soft set and interval set together. The organization of this paper is as follows. In Section 2 we present the correlative notions of interval sets. Section 3 deals with the algebraic structures of soft interval sets. The concept of soft interval sets is introduced by combining soft set and interval set. Some operations on soft interval sets are proposed and the lattice structures of soft interval sets are established. The soft quotient algebras are presented based the soft equality relations between soft interval sets. A soft interval set based decision making problem is examined in Section 4. We have some conclusions in the concluding Section 5.

\section{Interval sets}

Let $U$ be a set of objects. We consider the concepts with respect to objects in $U$. A concept, in the classical view, is defined by a pair of intension and extension. Although the extension of a concept is actually a subset of $U$, in many practical situations, a lack of knowledge makes us unable to specify this subset. Sometimes we can only provide a lower bound $A_{l}$ and an upper bound $A_{u}$. Any subset $A$ that lies between $A_{l}$ and $A_{u}$, namely, $A_{l} \subseteq A \subseteq A_{u}$ can be the actual extension of the concept. Based on these observations, $\mathrm{Yao}^{20}$ proposed the notion of interval sets.

Definition $1{ }^{20}$ Let $U$ be a non-empty set, called the universe, and $2^{U}$ be its power set. A subset of $2^{U}$ of the form

$$
\mathscr{A}=\left[A_{l}, A_{u}\right]=\left\{A \in 2^{U} ; A_{l} \subseteq A \subseteq A_{u}\right\}
$$

is called an interval set on $U$, where it is assumed $A_{l} \subseteq A_{u} . A_{l}$ and $A_{u}$ are called the lower bound and upper bound of $\mathscr{A}$ respectively.

In other words, an interval set on $U$ is a family of subsets of $U$. It is just an interval of the power set lattice $2^{U}$. The symbols $\in, \subseteq, \cap, \cup$ and - may be used in their usual set-theoretic sense to represent relationships between elements of $2^{U}$ and an interval set, and between different interval sets. Degenerate interval sets of the form $[A, A]$ are equivalent to ordinary sets. Thus, interval sets may be considered 
as an extension of elementary sets. In what follows, the set of all interval sets on $U$ is denoted by $I\left(2^{U}\right)$.

Interval set approach provides a tool for modeling and processing partially known concepts and for approximating undefinable or complex concepts. Operations on interval sets are defined by $\mathrm{Yao}^{20}$ based on the corresponding set-theoretic operations in a manner parallel to that used in defining operations on interval numbers. Thus, interval set algebra may be regarded as a counterpart of the interval number algebra proposed by Moore ${ }^{24}$.

Definition $2{ }^{20}$ Let $\mathscr{A}=\left[A_{1}, A_{2}\right]$ and $\mathscr{B}=\left[B_{1}, B_{2}\right]$ be two interval sets on $U$. The interval-set intersection, union, and difference of $\mathscr{A}$ and $\mathscr{B}$ are defined as:

$$
\begin{aligned}
& \mathscr{A} \sqcap \mathscr{B}=\{A \cap B ; A \in \mathscr{A}, B \in \mathscr{B}\}, \\
& \mathscr{A} \sqcup \mathscr{B}=\{A \cup B ; A \in \mathscr{A}, B \in \mathscr{B}\}, \\
& \mathscr{A} \backslash \mathscr{B}=\{A-B ; A \in \mathscr{A}, B \in \mathscr{B}\} .
\end{aligned}
$$

Additionally, the interval-set complement of $\mathscr{A}$ is defined as:

$$
\neg \mathscr{A}=[U, U] \backslash \mathscr{A} \text {. }
$$

Interval-set intersection, union, difference and complement are closed operations on $I\left(2^{U}\right)$. Actually, for arbitrary interval sets $\mathscr{A}=\left[A_{1}, A_{2}\right]$ and $\mathscr{B}=\left[B_{1}, B_{2}\right]$ on $U$, we have ${ }^{20}$ :

$$
\begin{aligned}
& \mathscr{A} \sqcap \mathscr{B}=\left[A_{1} \cap A_{2}, B_{1} \cap B_{2}\right], \\
& \mathscr{A} \sqcup \mathscr{B}=\left[A_{1} \cup A_{2}, B_{1} \cup B_{2}\right], \\
& \mathscr{A} \backslash \mathscr{B}=\left[A_{1}-A_{2}, B_{1}-B_{2}\right]=\mathscr{A} \sqcap \neg \mathscr{B}, \\
& \neg \mathscr{A}=\left[A_{2}^{c}, A_{1}^{c}\right] .
\end{aligned}
$$

The following list summarizes properties of interval sets ${ }^{25}$ : for $\mathscr{A}, \mathscr{B}, \mathscr{C} \in I\left(2^{U}\right)$,

(I1) Idempotent: $\mathscr{A} \sqcap \mathscr{A}=\mathscr{A}, \mathscr{A} \sqcup \mathscr{A}=\mathscr{A}$.

(I2) Commutativity: $\mathscr{A} \sqcap \mathscr{B}=\mathscr{B} \sqcap \mathscr{A}, \mathscr{A} \sqcup \mathscr{B}=$ $\mathscr{B} \sqcup \mathscr{A}$.

(I3) Associativity: $(\mathscr{A} \sqcap \mathscr{B}) \sqcap \mathscr{C}=\mathscr{A} \sqcap(\mathscr{B} \sqcap \mathscr{C})$, $(\mathscr{A} \sqcup \mathscr{B}) \sqcup \mathscr{C}=\mathscr{A} \sqcup(\mathscr{B} \sqcup \mathscr{C})$.

(I4) Distributivity: $\mathscr{A} \sqcap(\mathscr{B} \sqcup \mathscr{C})=(\mathscr{A} \sqcap \mathscr{B}) \sqcup$ $(\mathscr{A} \sqcap \mathscr{C}), \mathscr{A} \sqcup(\mathscr{B} \sqcap \mathscr{C})=(\mathscr{A} \sqcup \mathscr{B}) \sqcap(\mathscr{A} \sqcup \mathscr{C})$.

(I5) Absorption: $\mathscr{A} \sqcap(\mathscr{A} \sqcup \mathscr{B})=\mathscr{A}, \mathscr{A} \sqcup(\mathscr{A} \sqcap$ $\mathscr{B})=\mathscr{A}$.

(I6) De Morgan's laws: $\neg(\mathscr{A} \sqcap \mathscr{B})=\neg \mathscr{A} \sqcup \neg \mathscr{B}$, $\neg(\mathscr{A} \sqcup \mathscr{B})=\neg \mathscr{A} \sqcap \neg \mathscr{A}$.

(I7) Double negation law: $\neg \neg \mathscr{A}=\mathscr{A}$.

Hence, $\left(I\left(2^{U}\right), \sqcup, \sqcap\right)$ is a distributive lattice ${ }^{25}$. The order relation $\sqsubseteq$ on $I\left(2^{U}\right)$ can be defined by:
$\left[A_{1}, A_{2}\right] \sqsubseteq\left[B_{1}, B_{2}\right]$ if and only if $A_{1} \subseteq B_{1}$ and $A_{2} \subseteq B_{2}$.

In addition, $[U, U]$ and $[\emptyset, \emptyset]$ are the greatest element and the least element in $I\left(2^{U}\right)$, respectively.

It is worth noticing that, for an interval set $\mathscr{A}=$ $\left[A_{1}, A_{2}\right], \mathscr{A} \sqcap \neg \mathscr{A}$ is not necessarily equal to $[\emptyset, \emptyset]$, and $\mathscr{A} \sqcup \neg \mathscr{A}$ is not necessarily equal to $[U, U]$. Actually, we have $\mathscr{A} \sqcap \neg \mathscr{A}=\left[\emptyset, A_{2}-A_{1}\right], \mathscr{A} \sqcup \neg \mathscr{A}=$ $\left[\left(A_{2}-A_{1}\right)^{c}, U\right]$. Hence $\emptyset \in \mathscr{A} \sqcap \neg \mathscr{A}, U \in \mathscr{A} \sqcup \neg \mathscr{A}$.

\section{Soft interval sets}

Soft set theory was initiated by Molodtsov as a mathematical tool for dealing with uncertainty. In this section, by introducing interval set into the theory of soft sets, we propose the concept of soft interval sets and investigate its algebraic structures.

\subsection{Concept of and operations on soft interval sets}

Let $U$ be an initial universe set and $E$ the set of all possible parameters under consideration with respect to $U$. Usually, parameters are attributes, characteristics, or properties of objects in $U$. $(U, E)$ is called a soft universe. Molodtsov defined the notion of a soft set in the following way:

Definition $3{ }^{3} A$ pair $(F, A)$ is called a soft set over $U$, where $A \subseteq E$ and $F$ is a mapping given by $F$ : $A \rightarrow 2^{U}$.

Example 1 (1) Suppose that there are five houses in the universe $U$ given by $U=\left\{h_{1}, h_{2}, h_{3}, h_{4}, h_{5}\right\}$ and $E=\left\{e_{1}, e_{2}, e_{3}, e_{4}, e_{5}, e_{6}\right\}$ is the set of parameters. Where $e_{1}, e_{2}, e_{3}, e_{4}, e_{5}$ and $e_{6}$ stand for the parameters 'expensive', 'beautiful', 'in the green surroundings', 'in good repair', 'wooden', and 'modern' respectively.

In this case, to define a soft set means to point out expensive houses, beautiful houses, and so on. The soft set $(F, A)$ may describe the 'attractiveness of the houses' which Mr.X is going to buy. Suppose that $A=\left\{e_{1}, e_{2}, e_{3}, e_{4}\right\}$ is the set of parameters Mr.X concerned, $F\left(e_{1}\right)=\left\{h_{2}, h_{4}\right\}, F\left(e_{2}\right)=\left\{h_{1}, h_{3}\right\}, F\left(e_{3}\right)=$ $\left\{h_{3}, h_{4}, h_{5}\right\}$, and $F\left(e_{4}\right)=\left\{h_{1}, h_{5}\right\}$. Then the soft set $(F, A)$ is a parameterized family $\left\{F\left(e_{i}\right) ; 1 \leqslant i \leqslant 4\right\}$ of subsets of $U$ and give us a collection of approximate 
descriptions of an object. $F\left(e_{1}\right)=\left\{h_{2}, h_{4}\right\}$ means houses $h_{2}$ and $h_{4}$ are 'expensive'.

(2) Interval set may be considered as a special case of the soft set. Let $\mathscr{A}=\left[A_{1}, A_{2}\right]$ be an interval set on the universe $U$ and $C=A_{2}-A_{1}$. It follows that $\mathscr{A}=\left\{F(B) ; B \in 2^{C}\right\}$ where $F(B)=A_{1} \cup B$ for every $B \in 2^{C}$. Thus, the interval set $\mathscr{A}$ may be considered as the soft set $\left(F, 2^{C}\right)$.

By the definition, a soft set $(F, A)$ over $U$ is a parameterized family of subsets of $U$. For $e \in A, F(e)$ may be considered as the set of $e$-approximate elements of the soft set $(F, A)$. We notice that, in some real applications, $F(e)$ cannot be precisely defined because of the complexity of the practical problems. Maybe we can only provide a lower bound and an upper bound of $F(e)$. In this case, interval set is a appropriate substitution for crisp subset of the universe.

Definition 4 A pair $(F, A)$ is called a soft interval set over $U$, where $A \subseteq E$ and $F$ is a mapping given by $F: A \rightarrow I\left(2^{U}\right)$.

By this definition, a soft interval set over $U$ is a parameterized family of interval sets on $U$. Clearly, soft interval set is a generalization of soft set. In what follows, we denote by $S I\left(2^{U}\right)$ the set of all soft interval sets over $U$.

Example 2 We consider the soft universe $(U, E)$ given in Example $1(1) . \quad(F, A)$ is a soft interval set, where $A=\left\{e_{1}, e_{2}, e_{3}, e_{4}\right\}$ is the set of parameters we concerned and $F\left(e_{1}\right)=$ $\left[\left\{h_{2}, h_{4}\right\},\left\{h_{1}, h_{2}, h_{4}\right\}\right], \quad F\left(e_{2}\right)=\left[\left\{h_{1}, h_{3}\right\},\left\{h_{1}, h_{3}, h_{4}\right\}\right]$, $F\left(e_{3}\right)=\left[\left\{h_{3}, h_{4}, h_{5}\right\},\left\{h_{3}, h_{4}, h_{5}\right\}\right]$, and $F\left(e_{4}\right)=$ $\left[\left\{h_{5}\right\},\left\{h_{1}, h_{2}, h_{5}\right\}\right]$.

This soft interval set gives us a collection of approximate descriptions of an object. $F\left(e_{1}\right)=$ $\left[\left\{h_{2}, h_{4}\right\},\left\{h_{1}, h_{2}, h_{4}\right\}\right]$ means houses $h_{2}, h_{4} \in\left\{h_{2}, h_{4}\right\}$ are definitely expensive, $h_{1} \in\left\{h_{1}, h_{2}, h_{4}\right\}-\left\{h_{2}, h_{4}\right\}$ is possibly expensive, and $h_{3}, h_{5} \in U-\left\{h_{1}, h_{2}, h_{4}\right\}$ are not expensive.

Maji et al. ${ }^{7}$ and Irfan et al. ${ }^{8}$ introduced some operations on soft sets. Similarly, we introduce the operations on soft interval sets based on the operations on interval sets.

Definition 5 Let $(F, A)$ and $(G, B)$ be two soft interval sets over a common universe $U$.
(1) The extended union of $(F, A)$ and $(G, B)$, denoted by $(F, A) \cup_{e}(G, B)$, is the soft interval set $(H, C)$, where $C=A \cup B$, and $H$ is given by:

$$
H(a)= \begin{cases}F(a), & \text { if } a \in A-B, \\ G(a), & \text { if } a \in B-A, \\ F(a) \sqcup G(a), & \text { if } a \in A \cap B .\end{cases}
$$

(2) The extended intersection of $(F, A)$ and $(G, B)$, denoted by $(F, A) \cap_{e}(G, B)$, is the soft interval set $(H, C)$, where $C=A \cup B$, and $H$ ia given by:

$$
H(a)= \begin{cases}F(a), & \text { if } a \in A-B, \\ G(a), & \text { if } a \in B-A, \\ F(a) \sqcap G(a), & \text { if } a \in A \cap B .\end{cases}
$$

(3) The restricted union of $(F, A)$ and $(G, B)$, denoted by $(F, A) \cup_{r}(G, B)$, is the soft interval set $(H, C)$, where $C=A \cap B$, and $H(a)=F(a) \sqcup G(a)$ for every $a \in C$.

(4) The restricted intersection of $(F, A)$ and $(G, B)$, denoted by $(F, A) \cap_{r}(G, B)$, is the soft interval set $(H, C)$, where $C=A \cap B$, and $H(a)=F(a) \sqcap G(a)$ for every $a \in C$.

Definition $6(1)(F, A)$ is called a relative null soft interval set(with respect to the parameter set $A)$, denoted by $\emptyset_{A}$, if $F(e)=[\emptyset, \emptyset]$ for all $e \in A$.

(2) $(G, A)$ is called a relative whole soft interval set(with respect to the parameter set $A)$, denoted by $U_{A}$, if $F(e)=[U, U]$ for all $e \in A$.

Definition 7 The relative complement of a soft interval set $(F, A)$ is denoted by $(F, A)^{r}$ and is defined by $(F, A)^{r}=\left(F^{r}, A\right)$, where $F^{r}: A \rightarrow I\left(2^{U}\right)$ is a mapping given by $F^{r}(e)=\neg F(e)$ for all $e \in A$.

Clearly, $\left((F, A)^{r}\right)^{r}=(F, A)$ holds.

\subsection{Algebraic structures of soft interval sets}

Algebraic structures play a fundamental role in many fields of mathematics. Borzooei et al. ${ }^{26}$ discussed the lattice structures of some fuzzy algebraic systems. We know that an interval set on a universe $U$ is a bounded sublattice of the power set lattice $2^{U}$ and the set of all interval sets on $U$ forms a distributive lattice. In addition, Wang et al. ${ }^{27}$ proposed $G$-implication and $L$-implication operators on interval sets and constructed $G$-algebra and $M V$-algebra of interval sets. In this subsection, we consider the lattice structure of soft interval sets. 
Based on the order relation $\sqsubseteq$ on interval sets, we define the order relation $\subseteq$ on soft interval sets as follows: for arbitrary soft interval sets $(F, A)$ and $(G, B)$, $(F, A) \subseteq(G, B)$ if and only if $A \subseteq B$ and $F(a) \sqsubseteq G(a)$ for every $a \in A$.

We observe that this order relation is compatible with the operation on soft interval sets, i.e.,

$(F, A) \subseteq(G, B)$ if and only if $(F, A) \cup_{e}(G, B)=$ $(G, B)$.

In fact, let $(F, A) \cup_{e}(G, B)=(H, A \cup B)$. If $(F, A) \subseteq$ $(G, B)$, then $A \subseteq B$ and hence $A \cup B=B$. For each $a \in B$, if $a \notin A$, then $H(a)=G(a)$ by the definition; if $a \in A$, then $H(a)=F(a) \sqcup G(a)=G(a)$ by $F(a) \sqsubseteq G(a)$. Thus we have $(F, A) \cup_{e}(G, B)=(G, B)$. Conversely, we assume that $(F, A) \cup(G, B)=(G, B)$. It follows that $A \subseteq B$ and for each $a \in A$, by $F(a) \sqcup$ $G(a)=G(a)$ we conclude that $F(a) \sqsubseteq G(a)$ and hence $(F, A) \subseteq(G, B)$. Thus, the order relation $\subseteq$ on soft interval sets is just the order relation induced by $\cup_{e}$.

It is trivial to verify that $\left(S I\left(2^{U}\right), \subseteq\right)$ is a partially ordered sets. Actually, we have:

Theorem 1 (1) In $\left(S I\left(2^{U}\right), \subseteq\right)$, the least upper bound $(F, A) \vee(G, B)$ and greatest lower bound $(F, A) \wedge(G, B)$ of $(F, A)$ and $(G, B)$ can be explicitly computed by using the following formulas:

$$
\begin{aligned}
& (F, A) \vee(G, B)=(F, A) \cup_{e}(G, B), \\
& (F, A) \wedge(G, B)=(F, A) \cap_{r}(G, B) . \\
& (2)\left(S I\left(2^{U}\right), \cup_{e}, \cap_{r}\right) \text { is a bounded distributive lat- }
\end{aligned}
$$
tice.

Proof. (1) We prove that $(F, A) \vee(G, B)=(F, A) \cup_{e}$ $(G, B)$ holds. Suppose that $(F, A) \cup(G, B)=(H, A \cup$ $B)$. For every $a \in A$, if $a \notin B$, then $F(a)=H(a)$; if $a \in B$, then $F(a) \sqsubseteq F(a) \sqcup G(a)=H(a)$. We conclude that $(F, A) \subseteq(F, A) \cup(G, B)$. Similarly, we have $(G, B) \subseteq(F, A) \cup_{e}(G, B)$. Hence, $(F, A) \cup_{e}(G, B)$ is an upper bound of $(F, A)$ and $(G, B)$.

Let $(M, C)$ be an upper bound of $(F, A)$ and $(G, B)$, i.e., $(F, A) \subseteq(M, C),(G, B) \subseteq(M, C)$. It follows that $A \subseteq C, B \subseteq C$ and hence $A \cup B \subseteq C$. For every $a \in A \cup B$, if $a \in A-B$, then $H(a)=F(a) \sqsubseteq M(a)$; if $a \in B-A$, then $H(a)=G(a) \sqsubseteq M(a)$; if $a \in A \cap B$, then $H(a)=F(a) \sqcup G(a) \sqsubseteq M(a)$ by $F(a) \sqsubseteq M(a)$ and $G(a) \sqsubseteq M(a)$. We conclude that $(F, A) \cup_{e}(G, B) \subseteq$ $(M, C)$. That is, $(F, A) \cup(G, B)$ is the least upper bound of $(F, A)$ and $(G, B)$.
$(F, A) \wedge(G, B)=(F, A) \cap_{r}(G, B)$ can be proved similarly.

(2) By (1) we know that $\left(S I\left(2^{U}\right), \cup_{e}, \cap_{r}\right)$ is a lattice. Trivially, $\emptyset_{\emptyset}$ and $U_{E}$ are the least and greatest elements in $S I\left(2^{U}\right)$ respectively. We prove that the following distributive law

$(F, A) \cup_{e}\left((G, B) \cap_{r}(H, C)\right)$

$=\left((F, A) \cup_{e}(G, B)\right) \cap_{r}\left((F, A) \cup_{e}(H, C)\right)$

holds for all $(F, A),(G, B),(H, C) \in S I\left(2^{U}\right)$. Suppose that

$$
\begin{aligned}
& (F, A) \cup_{e}\left((G, B) \cap_{r}(H, C)\right)=(K, A \cup(B \cap C)), \\
& \left((F, A) \cup_{e}(G, B)\right) \cap_{r}\left((F, A) \cup_{e}(H, C)\right) \\
& =(L,(A \cup B) \cap(A \cup C))=(L, A \cup(B \cap C)) .
\end{aligned}
$$

For each $a \in A \cup(B \cap C)$,

(a) if $a \notin A$, then $a \in B$ and $a \in C$, it follows that $K(a)=G(a) \sqcap H(a)=L(a)$;

(b) if $a \in A, a \notin B, a \notin C$, then $K(a)=F(a)=$ $F(a) \sqcap F(a)=L(a)$;

(c) if $a \in A, a \in B, a \notin C$, then $K(a)=F(a)=$ $(F(a) \sqcup G(a)) \sqcap F(a)=L(a)$;

(d) if $a \in A, a \notin B, a \in C$, then $K(a)=F(a)=$ $F(a) \sqcap(F(a) \sqcup H(a))=L(a)$;

(e) if $a \in A, a \in B, a \in C$, then $K(a)=F(a) \sqcup$ $(G(a) \sqcap H(a))=(F(a) \sqcup G(a)) \sqcap(F(a) \sqcup H(a))=L(a)$.

Hence $(F, A) \cup_{e}\left((G, B) \cap_{r}(H, C)\right)=\left((F, A) \cup_{e}\right.$ $(G, B)) \cap_{r}\left((F, A) \cup_{e}(H, C)\right)$.

We consider soft interval sets over a definite parameter set. Let $A \subseteq E$ and

$$
S I_{A}\left(2^{U}\right)=\left\{(F, A) ; F: A \rightarrow I\left(2^{U}\right)\right\}
$$

be the set of soft interval sets over the universe $U$ and the parameter set $A$. It is trivial to verify that $(F, A) \cup_{e}(G, A),(F, A) \cap_{r}(G, A) \in S I_{A}\left(2^{U}\right)$ for all $(F, A),(G, A) \in S I_{A}\left(2^{U}\right)$. Hence we have the following Corollary.

Corollary $1\left(S I_{A}\left(2^{U}\right), \cup_{e}, \cap_{r}\right)$ is a sublattice of $\left(S I\left(2^{U}\right), \cup_{e}, \cap_{r}\right)$.

In $\left(S I_{A}\left(2^{U}\right), \cup_{e}, \cap_{r}\right), U_{A}$ and $\emptyset_{A}$ are the greatest element and the least element respectively.

Now, we consider the algebraic structure of soft interval sets with respect to operations $\cup_{r}$ and $\cap_{e}$. We define the order relation $\subseteq^{\prime}$ on soft interval sets as follows: for arbitrary soft interval sets $(F, A)$ and $(G, B),(F, A) \subseteq^{\prime}(G, B)$ if and only if $A \supseteq B$ and $F(b) \sqsubseteq G(b)$ for every $b \in B$. 
It is easy to see that $\left(S I\left(2^{U}\right), \subseteq^{\prime}\right)$ is a partially ordered sets.

Theorem 2 (1) In $\left(S I\left(2^{U}\right), \subseteq^{\prime}\right)$, the least upper bound $(F, A) \vee(G, B)$ and greatest lower bound $(F, A) \wedge(G, B)$ of $(F, A)$ and $(G, B)$ can be explicitly computed by using the following formulas:

$(F, A) \vee(G, B)=(F, A) \cup_{r}(G, B)$,

$(F, A) \wedge(G, B)=(F, A) \cap_{e}(G, B)$.

(2) $\left(S I\left(2^{U}\right), \cup_{r}, \cap_{e}\right)$ is a bounded distributive lattice.

Proof. (1) We prove $(F, A) \vee(G, B)=(F, A) \cup_{r}$ $(G, B)$. Suppose that $(F, A) \cup_{r}(G, B)=(H, A \cap B)$. It follows that $A \cap B \subseteq A$ and $F(a) \sqsubseteq F(a) \sqcup G(a)=H(a)$ for all $a \in A \cap B$. We conclude that $(F, A) \subseteq^{\prime}(F, A) \cup r$ $(G, B)$. Similarly, we have $(G, B) \subseteq^{\prime}(F, A) \cup_{r}(G, B)$. Hence, $(F, A) \cup_{r}(G, B)$ is an upper bound of $(F, A)$ and $(G, B)$ with respect to $\subseteq^{\prime}$.

Let $(F, A) \subseteq^{\prime}(M, C),(G, B) \subseteq^{\prime}(M, C)$. It follows that $C \subseteq A, C \subseteq B$ and hence $C \subseteq A \cap B$. For every $a \in C$, we have $F(a) \sqsubseteq M(a), G(a) \sqsubseteq M(a)$ and consequently $H(a)=F(a) \sqcup G(a) \sqsubseteq M(a)$. We conclude that $(F, A) \cup_{r}(G, B) \subseteq^{\prime}(M, C)$. That is, $(F, A) \cup_{r}(G, B)$ is the least upper bound of $(F, A)$ and $(G, B)$.

$(F, A) \wedge(G, B)=(F, A) \cap_{e}(G, B)$ can be proved similarly.

(2) By the definition of $\subseteq^{\prime}$ we know that $\emptyset_{E}$ and $\emptyset_{\emptyset}$ are the least and greatest elements in $S I\left(2^{U}\right)$ with respect to $\subseteq^{\prime}$, respectively. We prove that the following distributive law

$$
\begin{aligned}
& (F, A) \cup_{r}\left((G, B) \cap_{e}(H, C)\right) \\
& =\left((F, A) \cup_{r}(G, B)\right) \cap_{e}\left((F, A) \cup_{r}(H, C)\right) \\
& \text { holds for all }(F, A),(G, B),(H, C) \in S I\left(2^{U}\right) \\
& \text { Suppose that } \\
& (F, A) \cup_{r}\left((G, B) \cap_{e}(H, C)\right)=(K, A \cap(B \cup C)), \\
& \left((F, A) \cup_{r}(G, B)\right) \cap_{e}\left((F, A) \cup_{r}(H, C)\right) \\
& =(L,(A \cap B) \cup(A \cap C))=(L, A \cap(B \cup C))
\end{aligned}
$$

For each $a \in A \cap(B \cup C)$, we have $a \in A$ and $a \in B \cup C$.

(a) if $a \in B$ and $a \in C$, then $K(a)=F(a) \sqcup(G(a) \sqcap$ $H(a))=(F(a) \sqcup G(a)) \sqcap(F(a) \sqcup H(a))=L(a)$;

(b) if $a \notin B, a \in C$, then $K(a)=F(a) \sqcup H(a)=$ $L(a)$;

(c) if $a \in B, a \notin C$, then $K(a)=F(a) \sqcup G(a)=L(a)$.

Since $K$ and $L$ are indeed the same intervalset-valued mappings, we conclude that $(F, A) \cup_{r}$
$\left((G, B) \cap_{e}(H, C)\right)=\left((F, A) \cup_{r}(G, B)\right) \cap_{e}\left((F, A) \cup_{r}\right.$ $(H, C))$ as required.

Corollary $2\left(S I_{A}\left(2^{U}\right), \cup_{r}, \cap_{e}\right)$ is a sublattice of $\left(S I\left(2^{U}\right), \cup_{r}, \cap_{e}\right)$.

In $\left(S I_{A}\left(2^{U}\right), \cup_{e}, \cap_{r}\right), \emptyset_{A}$ and $U_{A}$ are the least element and the greatest element respectively. For every $(F, A),(G, A) \in S I_{A}\left(2^{U}\right)$, we have $(F, A) \subseteq(G, A)$ if and only if $(F, A) \subseteq^{\prime}(G, A)$. It follows that $\left(S I_{A}\left(2^{U}\right), \cup_{e}, \cap_{r}\right)$ and $\left(S I_{A}\left(2^{U}\right), \cup_{r}, \cap_{e}\right)$ are the same lattices.

The following types of De Morgan's laws hold in soft interval set theory.

Theorem 3 Let $(F, A)$ and $(G, B)$ be two soft interval sets over the same universe $U$ such that $A \cap B \neq$ $\emptyset$. Then

$$
\begin{aligned}
& (1)\left((F, A) \cup_{r}(G, B)\right)^{r}=(F, A)^{r} \cap_{r}(G, B)^{r}, \\
& (2)\left((F, A) \cap_{r}(G, B)\right)^{r}=(F, A)^{r} \cup_{r}(G, B)^{r}, \\
& (3)\left((F, A) \cup_{e}(G, B)\right)^{r}=(F, A)^{r} \cap_{e}(G, B)^{r}, \\
& \text { (4) }\left((F, A) \cap_{e}(G, B)\right)^{r}=(F, A)^{r} \cup_{e}(G, B)^{r} .
\end{aligned}
$$

Proof. (1) Let $(F, A) \cup_{r}(G, B)=(H, A \cap B)$, $(F, A)^{r} \cap_{r}(G, B)^{r}=(K, A \cap B)$. It follows that $\left((F, A) \cup_{r}(G, B)\right)^{r}=\left(H^{r}, A \cap B\right)$. For all $a \in A \cap B$, we have $H^{r}(a)=\neg H(a)=\neg(F(a) \sqcup G(a))=\neg F(a) \sqcap$ $\neg G(a)=F^{r}(a) \sqcap G^{r}(a)=K(a)$, and consequently $\left((F, A) \cup_{r}(G, B)\right)^{r}=(F, A)^{r} \cap_{r}(G, B)^{r}$.

(2) By (1) we have $\left((F, A)^{r} \cup_{r}(G, B)^{r}\right)^{r}=$ $\left((F, A)^{r}\right)^{r} \cap_{r}\left((G, B)^{r}\right)^{r}=(F, A) \cap_{r}(G, B), \quad$ and hence $\left((F, A) \cap_{r}(G, B)\right)^{r}=\left(\left((F, A)^{r} \cup_{r}(G, B)^{r}\right)^{r}\right)^{r}=$ $(F, A)^{r} \cup_{r}(G, B)^{r}$.

(3) Let $(F, A) \cup_{e}(G, B)=(H, A \cup B),(F, A)^{r} \cap_{e}$ $(G, B)^{r}=(K, A \cup B)$. It follows that $((F, A) \cup e$ $(G, B))^{r}=\left(H^{r}, A \cup B\right)$. For all $a \in A \cup B$,

(a) if $a \in A-B$, then $H^{r}(a)=\neg H(a)=\neg F(a)=$ $K(a)$;

(b) if $a \in B-A$, then $H^{r}(a)=\neg H(a)=\neg G(a)=$ $K(a)$

(c) if $a \in A \cap B$, then $H^{r}(a)=\neg H(a)=\neg(F(a) \sqcup$ $G(a))=(\neg F(a)) \sqcap(\neg G(a))=K(a)$.

Hence we have $\left((F, A) \cup_{e}(G, B)\right)^{r}=(F, A)^{r} \cap_{e}$ $(G, B)^{r}$.

(4) By (3) we have $\left((F, A)^{r} \cup_{e}(G, B)^{r}\right)^{r}=$ $\left((F, A)^{r}\right)^{r} \cap_{e}\left((G, B)^{r}\right)^{r}=(F, A) \cap_{e}(G, B)$. It follows that $\left((F, A) \cap_{e}(G, B)\right)^{r}=\left(\left((F, A)^{r} \cup_{e}(G, B)^{r}\right)^{r}\right)^{r}=$ $(F, A)^{r} \cup_{e}(G, B)^{r}$. 


\subsection{The soft equality relation on soft interval sets}

Based on the parameter analysis of soft set, Qin et al. ${ }^{9}$ introduced the notion of soft equality relation on soft sets. It is used to describe soft sets which are 'almost' equal. With this relation, all the soft sets on a definite universe can be partitioned to different classes in such a way that two soft sets will be partitioned to same class if they are soft equal. Furthermore, the soft equality relation can be used to construct soft quotient algebra. In this subsection, we follow the line of exploration in [9] and intend to generalize this relation to soft interval sets.

Definition 8 Let $(F, A),(G, B)$ be two soft interval sets over the universe $U .(F, A)$ is called soft equal to $(G, B)$, denoted by $(F, A) \approx_{S}(G, B)$, if for all $a \in A \cup$ $B, a \in A \cap B$ implies $F(a)=G(a), a \in A-B$ implies $F(a)=[\emptyset, \emptyset]$, and $a \in B-A$ implies $G(a)=[\emptyset, \emptyset]$.

Example 3 We consider the soft interval set $(F, A)$ given in Example 2. Let $(G, B)$ be a soft interval set, where $B=\left\{e_{1}, e_{2}, e_{3}, e_{4}, e_{5}\right\}, G\left(e_{i}\right)=F\left(e_{i}\right)$ for every $1 \leqslant i \leqslant 4$, and $G\left(e_{5}\right)=[\emptyset, \emptyset]$. Then $(F, A) \approx_{S}(G, B)$.

Theorem 4 Let $(F, A)$ and $(G, B)$ be two soft interval sets over the universe $U$. Then $(F, A) \approx_{S}(G, B)$ if and only if $(F, A) \cup_{e}(G, B) \approx_{S}(F, A) \cap_{r}(G, B)$.

Proof. Let $(F, A) \cup_{e}(G, B)=(H, A \cup B)$ and $(F, A) \cap_{r}$ $(G, B)=(T, A \cap B)$.

Suppose that $(F, A) \approx_{S}(G, B)$. For all $a \in A \cap B$, by Definition 8 , we have $F(a)=G(a)$, and hence $H(a)=F(a) \sqcup G(a)=F(a) \sqcap G(a)=T(a)$. For all $a \in A \cup B-A \cap B$, (a)if $a \in A-B$, then $F(a)=[\emptyset, \emptyset]$ and hence $H(a)=F(a)=[\emptyset, \emptyset]$; (b)if $a \in B-A$, then $G(a)=[\emptyset, \emptyset]$ and hence $H(a)=G(a)=[\emptyset, \emptyset]$. Consequently, $(F, A) \cup_{e}(G, B) \approx_{S}(F, A) \cap_{r}(G, B)$.

Conversely, suppose that $(F, A) \cup_{e}(G, B) \approx_{S}$ $(F, A) \cap_{r}(G, B)$. For all $a \in A \cap B$, we have $F(a) \sqcup$ $G(a)=F(a) \sqcap G(a)$ and hence $F(a)=G(a)$. For all $a \in A-B$, it follows that $a \in A \cup B, a \notin A \cap B$, and hence $F(a)=H(a)=[\emptyset, \emptyset]$. For all $a \in B-$ $A, G(a)=[\emptyset, \emptyset]$ can be proved similarly. Hence $(F, A) \approx_{S}(G, B)$.

Theorem 5 Let $(F, A),(G, B)$ be two soft sets over the universe $U$.
(1) $(F, A) \approx_{S}(G, B)$ if and only if $(F, A) \cup_{r}$ $(G, B) \approx_{S}(F, A) \cap_{e}(G, B)$.

(2) $(F, A) \approx_{S}(G, B)$ implies $(F, A) \cup_{e}(G, B)=$ $(F, A) \cap_{e}(G, B),(F, A) \cup_{r}(G, B)=(F, A) \cap_{r}(G, B)$.

The proof is similar to that of Theorem 4 and we omit it.

Theorem $6 \approx_{S}$ is a congruence relation on $S I\left(2^{U}\right)$ with respect to operations $\cap_{r}$ and $\cup_{e}$, i.e.,

$(1) \approx_{S}$ is an equivalence relation;

(2) $(F, A) \approx_{S}(G, B)$ and $(H, C) \approx_{S}(L, D)$ imply $(F, A) \cap_{r}(H, C) \approx_{S}(G, B) \cap_{r}(L, D)$ and $(F, A) \cup_{e}$ $(H, C) \approx_{S}(G, B) \cup_{e}(L, D)$.

Proof. (1) It is trivial to verify that $\approx_{S}$ is reflexive and symmetric.

Suppose that $(F, A) \approx_{S}(G, B)$ and $(G, B) \approx_{S}$ $(H, C)$. For all $e \in A \cap C$, if $e \in B$, then $e \in A \cap B$ and $e \in B \cap C$, it follows that $F(e)=G(e)=H(e)$; if $e \notin B$, then $e \in A-B$ and $e \in C-B$, it follows that $F(e)=[\emptyset, \emptyset]=H(e)$.

For all $e \in A-C$, it follows that $e \in A$ and $e \notin C$. If $e \in B$, then $e \in A \cap B$ and $e \in B-C$. Consequently, $F(e)=G(e)=[\emptyset, \emptyset]$; if $e \notin B$, then $e \in A-B$ and $F(e)=[\emptyset, \emptyset]$.

For all $e \in C-A, H(e)=[\emptyset, \emptyset]$ can be proved similarly. Hence $(F, A) \approx_{S}(H, C)$. We conclude that $\approx_{S}$ is a transitive relation as required.

(2) Suppose that $(F, A) \approx_{S}(G, B)$ and $(H, C) \approx_{S}$ $(L, D)$. Let $(F, A) \cap_{r}(H, C)=\left(M_{1}, A \cap C\right),(G, B) \cap r$ $(L, D)=\left(M_{2}, B \cap D\right)$.

(a) If $e \in(A \cap C) \cap(B \cap D)$, then $e \in(A \cap B)$ and $e \in(C \cap D)$. It follows that $F(e)=G(e), H(e)=L(e)$ and hence

$M_{1}(e)=F(e) \sqcap H(e)=G(e) \sqcap L(e)=M_{2}(e)$.

(b) If $e \in(A \cap C)-(B \cap D)$, then $e \in A, e \in C$ and $e \notin B \cap D$. It follows that $e \notin B$ or $e \notin D$. If $e \notin B$, then $e \in A-B$ and hence $F(e)=[\emptyset, \emptyset]$. Consequently, $M_{1}(e)=F(e) \sqcap H(e)=[\emptyset, \emptyset]$. If $e \notin D$, then $e \in C-D$ and hence $H(e)=[\emptyset, \emptyset]$. It follows that $M_{1}(e)=F(e) \sqcap H(e)=[\emptyset, \emptyset]$.

(c) If $e \in(B \cap D)-(A \cap C)$, then $M_{2}(e)=[\emptyset, \emptyset]$ can be proved similarly.

We conclude that $(F, A) \cap_{r}(H, C) \approx_{S}(G, B) \cap_{r}$ $(L, D)$.

Let $(F, A) \cup_{e}(H, C)=\left(T_{1}, A \cup C\right), \quad(G, B) \cup_{e}$ $(L, D)=\left(T_{2}, B \cup D\right)$. 
For all $e \in(A \cup C) \cap(B \cup D)$, we have $e \in A \cup C$ and $e \in B \cup D$. Without losing of generality, we suppose that $e \in A$ and $e \in D$.

(a) If $e \in B$ and $e \in C$, then $e \in A \cap B$ and $e \in C \cap D$. It follows that $F(e)=G(e), H(e)=L(e)$ and hence $T_{1}(e)=F(e) \sqcup H(e)=G(e) \sqcup L(e)=T_{2}(e)$.

(b) If $e \notin B$ and $e \in C$, then $e \in A-B$ and $e \in C \cap D$. It follows that $F(e)=[\emptyset, \emptyset], H(e)=L(e)$ and hence $T_{1}(e)=F(e) \sqcup H(e)=H(e)=L(e)=T_{2}(e)$.

(c) If $e \in B$ and $e \notin C$, then $e \in A \cap B$ and $e \in D-C$. It follows that $F(e)=G(e), L(e)=[\emptyset, \emptyset]$ and hence $T_{1}(e)=F(e)=G(e)=G(e) \sqcup L(e)=T_{2}(e)$.

(d) If $e \notin B$ and $e \notin C$, then $e \in A-B$ and $e \in D-C$. It follows that $F(e)=[\emptyset, \emptyset], L(e)=[\emptyset, \emptyset]$ and hence $T_{1}(e)=F(e)=[\emptyset, \emptyset]=L(e)=T_{2}(e)$.

For all $e \in A \cup C-B \cup D$, we have $e \in A \cup C, e \notin B$ and $e \notin D$.

(a) If $e \in A$ and $e \in C$, then $e \in A-B$ and $e \in$ $C-D$. It follows that $F(e)=H(e)=[\emptyset, \emptyset]$, and hence $T_{1}(e)=F(e) \sqcup H(e)=[\emptyset, \emptyset]$.

(b) If $e \in A$ and $e \notin C$, then $e \in A-B$. It follows that $F(e)=[\emptyset, \emptyset]$, and hence $T_{1}(e)=F(e)=[\emptyset, \emptyset]$.

(c) If $e \notin A$ and $e \in C$, then $e \in C-D$. It follows that $H(e)=[\emptyset, \emptyset]$, and hence $T_{1}(e)=H(e)=[\emptyset, \emptyset]$.

For all $e \in B \cup D-A \cup C, T_{2}(e)=[\emptyset, \emptyset]$ can be proved similarly.

Hence $(F, A) \cup_{e}(H, C) \approx_{S}(G, B) \cup_{e}(L, D)$.

Let $(F, A)_{\approx_{S}}=\left\{(G, B) ;(G, B) \approx_{S}(F, A)\right\}$ be the congruence class including $(F, A)$ and $S I\left(2^{U}\right) / \approx_{S}=$ $\left\{(F, A)_{\approx S} ;(F, A) \in S I\left(2^{U}\right)\right\}$ be the set of all congruence classes. We define operations $\cup_{S}$ and $\cap_{S}$ on $S I\left(2^{U}\right) / \approx_{S}$ as follows:

$$
\begin{aligned}
& (F, A)_{\approx_{S}} \cup_{S}(G, B)_{\approx_{S}}=\left((F, A) \cup_{e}(G, B)\right)_{\approx_{S}}, \\
& (F, A)_{\approx_{S}} \cap_{S}(G, B)_{\approx_{S}}=\left((F, A) \cap_{r}(G, B)\right)_{\approx_{S}} .
\end{aligned}
$$

These two operations are well defined by Theorem 6 . We call $\left(S I\left(2^{U}\right) / \approx_{S}, \cup_{S}, \cap_{S}\right)$ the soft quotient algebra with respect to $\approx_{S}$. It is routine to verify that $\left(S I\left(2^{U}\right) / \approx_{S}, \cup_{S}, \cap_{S}\right)$ is a distributive lattice.

Theorem $7\left(S I\left(2^{U}\right) / \approx_{S}, \cup_{S}, \cap_{S}\right)$ and $\left(S I_{E}\left(2^{U}\right), \cup_{e}, \cap_{r}\right)$ are isomorphic.

Proof. Let $\varphi: S I_{E}\left(2^{U}\right) \rightarrow S I\left(2^{U}\right) / \approx_{S}$ be defined as $\varphi(F, E)=(F, E)_{\approx S}$ for every $(F, E) \in S I_{E}\left(2^{U}\right)$.
(1) If $\left(F_{1}, E\right),\left(F_{2}, E\right) \in S I_{E}\left(2^{U}\right)$ and $\left(F_{1}, E\right) \neq$ $\left(F_{2}, E\right)$, then there exists $a \in E$ such that $F_{1}(a) \neq$ $F_{2}(a)$, and consequently $\left(F_{1}, E\right)_{\approx s} \neq\left(F_{2}, E\right)_{\approx s}$.

(2) Let $(F, A)_{\approx_{s}} \in S I\left(2^{U}\right) / \approx_{S}$. We define $\left(F^{\prime}, E\right) \in S I_{E}\left(2^{U}\right)$ as follows: $F^{\prime}(a)=[\emptyset, \emptyset]$ for each $a \in E-A$ and $F^{\prime}(a)=F(a)$ for each $a \in A$. Hence $\left(F^{\prime}, E\right) \approx_{S}(F, A)$ and $\varphi\left(F^{\prime}, E\right)=\left(F^{\prime}, E\right)_{\approx s}=(F, A)_{\approx s}$.

By (1) and (2), we conclude that $\varphi$ is a one-toone mapping.

(3) For every $\left(F_{1}, E\right),\left(F_{2}, E\right) \in S I_{E}\left(2^{U}\right)$, we have: $\varphi\left(\left(F_{1}, E\right) \cup_{e}\left(F_{2}, E\right)\right)=\left(\left(F_{1}, E\right) \cup_{e}\left(F_{2}, E\right)\right)_{\approx s}$ $=\left(F_{1}, E\right)_{\approx_{S}} \cup_{S}\left(F_{2}, E\right)_{\approx S}=\varphi\left(F_{1}, E\right) \cup_{S} \varphi\left(F_{2}, E\right)$, $\varphi\left(\left(F_{1}, E\right) \cap_{r}\left(F_{2}, E\right)\right)=\left(\left(F_{1}, E\right) \cap_{r}\left(F_{2}, E\right)\right)_{\approx s}$ $=\left(F_{1}, E\right)_{\approx_{S}} \cap_{S}\left(F_{2}, E\right)_{\approx_{S}}=\varphi\left(F_{1}, E\right) \cap_{S} \varphi\left(F_{2}, E\right)$.

We conclude that $\varphi$ is an isomorphism, $\left(S I\left(2^{U}\right) / \approx_{S}, \cup_{S}, \cap_{S}\right)$ and $\left(S I_{E}\left(2^{U}\right), \cup_{e}, \cap_{r}\right)$ are isomorphic.

In order to establish the soft quotient algebra with respect to operations $\cup_{r}$ and $\cap_{e}$, we discuss another kind of soft equality relation $\approx S$.

Definition 9 Let $(F, A),(G, B) \in S I\left(2^{U}\right) . \quad(F, A) \approx^{S}$ $(G, B)$ if for all $e \in A \cup B, e \in A \cap B$ implies $F(e)=$ $G(e), e \in A-B$ implies $F(e)=[U, U]$, and $e \in B-A$ implies $G(e)=[U, U]$.

Theorem 8 Let $(F, A)$ and $(G, B)$ be two soft interval sets over the universe $U$. Then $(F, A) \approx^{S}(G, B)$ if and only if $(F, A)^{r} \approx_{S}(G, B)^{r}$.

Proof. Suppose that $(F, A) \approx^{S}(G, B)$. For all $e \in A \cap B$, by definition, we have $F(e)=G(e)$, and hence $F^{r}(e)=\neg F(e)=G^{r}(e)$. For all $e \in A-B$, we have $F(e)=[U, U]$ and hence $F^{r}(e)=[\emptyset, \emptyset]$. Similarly, $G^{r}(e)=[\emptyset, \emptyset]$ for all $e \in B-A$. We conclude that $(F, A)^{r} \approx_{S}(G, B)^{r}$ as required.

Conversely, suppose that $(F, A)^{r} \approx_{S}(G, B)^{r}$. For all $e \in A \cap B$, we have $F^{r}(e)=G^{r}(e)$, and hence $F(e)=G(e)$. For all $e \in A-B$, we have $F^{r}(e)=[\emptyset, \emptyset]$ and hence $F(e)=[U, U]$. Similarly, $G(e)=[U, U]$ for all $e \in B-A$. Hence $(F, A) \approx^{S}(G, B)$.

Theorem $9 \approx^{S}$ is a congruence relation with respect to operations $\cup_{r}$ and $\cap_{e}$.

The proof of this Theorem is similar to that of Theorem 6.

Let $(F, A)_{\approx S}=\left\{(G, B) ;(G, B) \approx^{S}(F, A)\right\}$ be the congruence class (with respect to $\approx^{S}$ ) including 
$(F, A)$ and $S I\left(2^{U}\right) / \approx^{S}=\left\{(F, A)_{\approx^{S}} ;(F, A) \in S I\left(2^{U}\right)\right\}$. We define operations $\cup^{S}$ and $\cap^{S}$ on $S I\left(2^{U}\right) / \approx^{S}$ as follows:

$$
\begin{aligned}
& (F, A)_{\approx} \cup^{S}(G, B)_{\approx S}=\left((F, A) \cup_{r}(G, B)\right)_{\approx S}, \\
& (F, A)_{\approx S} \cap^{S}(G, B)_{\approx S}=\left((F, A) \cap_{e}(G, B)\right)_{\approx S} .
\end{aligned}
$$

We call $\left(S I\left(2^{U}\right) / \approx^{S}, \cup^{S}, \cap^{S}\right)$ the soft quotient algebra with respect to $\approx^{S}$. It is routine to verify that $\left(S I\left(2^{U}\right) / \approx^{S}, \cup^{S}, \cap^{S}\right)$ is a distributive lattice.

\section{Applications to decision making problems}

Maji et al. ${ }^{4}$ proposed tabular representation of soft set to deal with soft set based decision making problems. We can represent soft interval set similarly in a tabular form. Let $(F, A)$ be a soft interval set, $a \in A$ and $F(a)=\left[F(a)_{-}, F(a)_{+}\right]$. By intuitionistic semantics of soft interval set, we know that elements in $F(a)_{-}$belong definitely to $F(a)$, elements in $U-F(a)_{+}$does not belong to $F(a)$, while elements in $F(a)_{+}-F(a)_{-}$are possibly belong to $F(a)$. Following the idea of incomplete information system, we represent soft interval set $(F, A)$ in a binary table and its entries $h_{i j}$ are defined as: $h_{i} \in F(a)_{-}$implies $h_{i j}=1 ; h_{i} \in F(a)_{+}-F(a)_{-}$implies $h_{i j}=*$, and $h_{i} \in U-F(a)_{+}$implies $h_{i j}=0 . h_{i j}=*$ means we are not sure whether $h_{i} \in F(a)$ or not.

The tabular representation of the soft interval set $(F, A)$ given in Example 2 is as in Table 1.

Table 1. The tabular representation of $(F, A)$

\begin{tabular}{ccccc}
\hline & $e_{1}$ & $e_{2}$ & $e_{3}$ & $e_{4}$ \\
\hline$h_{1}$ & $*$ & 1 & 0 & $*$ \\
$h_{2}$ & 1 & 0 & 0 & $*$ \\
$h_{3}$ & 0 & 1 & 1 & 0 \\
$h_{4}$ & 1 & $*$ & 1 & 0 \\
$h_{5}$ & 0 & 0 & 1 & 1 \\
\hline
\end{tabular}

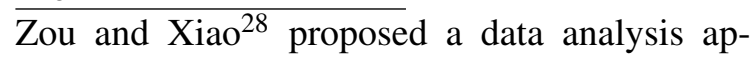
proach to soft set under incomplete information. The incomplete data will be predicted based on the method of average probability. Suppose $p_{a}$ stands for probability that an object in $F(a)_{+}-F(a)_{-}$belongs to $F(a)$, it can be defined by 28

$$
p_{a}=\frac{\left|F(a)_{-}\right|}{\left|F(a)_{-}\right|+\left|U-F(a)_{+}\right|}, a \in A .
$$

We may take $p_{a}$ as the value of elements in $F(a)_{+}-F(a)_{-}$with respect to parameter $a$. By this definition, for the soft interval set in Table 1, we have $p_{e_{1}}=\frac{1}{2}, p_{e_{2}}=\frac{1}{2}, p_{e_{4}}=\frac{1}{3}$. The extended tabular representation of $(F, A)$ is as in Table 2 .

Table 2. The extended tabular representation of $(F, A)$

\begin{tabular}{ccccc}
\hline & $e_{1}$ & $e_{2}$ & $e_{3}$ & $e_{4}$ \\
\hline$h_{1}$ & $\frac{1}{2}$ & 1 & 0 & $\frac{1}{3}$ \\
$h_{2}$ & 1 & 0 & 0 & $\frac{1}{3}$ \\
$h_{3}$ & 0 & 1 & 1 & 0 \\
$h_{4}$ & 0 & $\frac{1}{2}$ & 1 & 0 \\
$h_{5}$ & 0 & 0 & 1 & 1 \\
\hline
\end{tabular}

In real decision making problems, the choice parameters may not be of equal importance. To cope with such problems, we can impose different weights to different decision parameters. Additionally, there may be some parameters which is 'negative' to optimal choice. So the weights can range from -1 to 1 , that is, the values domain of weights is the interval $[-1,1]$.

Maji et al. ${ }^{4}$ proposed an approach to weighted soft set based decision making problems. The choice value $c_{i}$ of object $h_{i}$ will be computed by:

$$
c_{i}=\sum_{j} w_{j} h_{i j},
$$

where $w_{j}$ is the weight of $e_{j}$. The object with the maximum choice value will be selected as the optimal alternative. In this decision making problem, we know that $e_{1}$ stands for 'expensive' and it is negative to the optimal choice. We assume that the weights of $e_{1}, e_{2}, e_{3}, e_{4}$ are $-0.4,0.6,0.4,0.9$, respectively. The choice values $c_{i}$ of objects $h_{i}$ can be computed as follows:

$$
\begin{aligned}
& c_{1}=(-0.4) \times \frac{1}{2}+0.6 \times 1+0.4 \times 0+0.9 \times \frac{1}{3}=0.7, \\
& c_{2}=(-0.4) \times 1+0.6 \times 0+0.4 \times 0+0.9 \times \frac{1}{3}=-0.1, \\
& c_{3}=(-0.4) \times 0+0.6 \times 1+0.4 \times 1+0.9 \times 0=1, \\
& c_{4}=(-0.4) \times 1+0.6 \times \frac{1}{2}+0.4 \times 1+0.9 \times 0=0.3, \\
& c_{5}=(-0.4) \times 0+0.6 \times 0+0.4 \times 1+0.9 \times 1=1.3 .
\end{aligned}
$$

Hence, the optimal decision is to select $h_{5}$. The extended tabular representation of $(F, A)$ with weights and choice values is given by Table 3 . 
Table 3. The extended tabular representation of $(F, A)$ with weights and choice values

\begin{tabular}{cccccc}
\hline & $e_{1,-0.4}$ & $e_{2,0.6}$ & $e_{3,0.4}$ & $e_{4,0.9}$ & choice-value \\
\hline$h_{1}$ & $\frac{1}{2}$ & 1 & 0 & $\frac{1}{3}$ & 0.7 \\
$h_{2}$ & 1 & 0 & 0 & $\frac{1}{3}$ & -0.1 \\
$h_{3}$ & 0 & 1 & 1 & 0 & 1 \\
$h_{4}$ & 1 & $\frac{1}{2}$ & 1 & 0 & 0.3 \\
$h_{5}$ & 0 & 0 & 1 & 1 & 1.3 \\
\hline
\end{tabular}

This is only a simple example to show the possibility of using this method for decision making problems which could be improved based on domain knowledge and other competing data analysis methods. We summarize the soft interval set based decision making approach presented in this section as the following algorithm:

Step 1: Input the soft interval set $(F, A)$ and place it in tabular form.

Step 2: Compute average probability $p_{a}$ for each parameter $a \in A$ and construct the extended tabular representation of $(F, A)$, where $p_{a}=\frac{\left|F(a)_{-}\right|}{\left|F(a)_{-}\right|+\left|U-F(a)_{+}\right|}$.

Step 3: Input the weights $w_{j}$ of parameters in $A$.

Step 4: Compute the choice values $c_{i}$ of $h_{i}$, where $c_{i}=\sum_{j} w_{j} h_{i j}$ and $h_{i j}$ are entries in extended tabular representation of $(F, A)$.

Step 5: The optimal decision is $h_{k}$ if $c_{k}=\max _{i} c_{i}$.

Step 6: If $k$ has more that one value then any one of $h_{k}$ may be chosen.

\section{Conclusions}

Soft set theory and interval set theory are all mathematical tools for dealing with uncertainty. They are closely related. This paper deals with the combination of soft set and interval set models and its application. The notion of soft interval sets is introduced and the algebraic structure of soft interval sets is investigated. Additionally, a soft interval set based decision making problem is examined.

This paper focuses on the theoretical study of soft interval sets. Because of limitation of spaces, only a numerical example is provided to illustrate the application method of soft interval sets. We think that soft interval set theory may be applied to the knowledge acquisition for interval-valued information systems, qualitative information representation and qualitative information reasoning. Based on this paper, we can further probe the applications of soft interval sets in practical fields. The combination of soft interval set and other competing data analysis models is another important and interesting issue to be addressed.

\section{Acknowledgment}

The authors are grateful to the referees for their constructive suggestions on this paper. This work has been supported by the National Natural Science Foundation of China (Grant No.61175055 and 61175044).

\section{References}

1. L. A. Zadeh, Fuzzy sets, Information and Control 8(1965)338-353.

2. Z. Pawlak, Rough sets, Int. J. Comput. Inf. Sci. 11(1982)341-356.

3. D. Molodtsov, Soft set theory-First results, Comput. Math. Appl. 37(1999)19-31.

4. P. K. Maji, A. R. Roy and R. Biswas, An application of soft sets in a decision making problem, Comput. Math. Appl. 44(2002)1077-1083.

5. D. G. Chen, E. C. C. Tsang, D. S. Yeung and X. Z. Wang, The paremeterization reduction of soft sets and its applications, Comput. Math. Appl. 49(2005)757763.

6. Z. Kong, L. Q. Gao, L. F. Wang and S. Li, The normal paremeter reduction of soft sets and its algorithm, Comput. Math. Appl. 56(2008)3029-3037.

7. P. K. Maji, R. Biswas and A. R. Roy, Soft set theory, Comput. Math. Appl. 45(2003)555-562.

8. M. Irfan Ali, F. Feng, X. Liu, W. K. Min and M. Shabir, On some new operations in soft set theory, Comput. Math. Appl. 57(2009)1547-1553.

9. K. Y. Qin and Z. Y. Hong, On soft equality, J. Comput. Appl. Math. 234(2010)1347-1355.

10. H. Aktas and N. Cagman, Soft sets and soft groups, Inform. Sci. 177(2007)2726-2735.

11. Y. B. Jun, Soft BCK/BCI-algebras, Comput. Math. Appl. 56(2008)1408-1413.

12. Y. B. Jun and C. H. Park, Applications of soft sets in ideal theory of BCK/BCI-algebras, Inform. Sci. 178(2008)2466-2475.

13. P. K. Maji, R. Biswas and A. R. Roy, Fuzzy soft sets, J. Fuzzy Math. 9(3)(2001)589-602.

14. A. R. Roy and P. K. Maji, A fuzzy soft set theoretic approach to decision making problems, J. Comput. Appl. Math. 203(2007)412-418. 
15. X. B. Yang, T. Y. Lin, J. Y. Yang, Y. Li and D. J. Yu, Combination of interval-valued fuzzy set and soft set, Comput. Math. Appl. 58(2009)521-527.

16. F. Feng, Y. B. Jun, X. Y. Liu and L. F. Li, An adjustable approach to fuzzy soft set based decision making, J. Comput. Appl. Math. 234(2010)10-20.

17. F. Feng, Y. M. Li and L. F. Violeta, Application of level soft sets in decision making based on interval-valued fuzzy soft sets, Comput. Math. Appl. 60(2010)1756-1767.

18. F. Feng, C. X. Li, B. Davvaz and M. I. Ali, Soft sets combined with fuzzy sets and rough sets: a tentative approach, Soft Computing 14(2010)899-911.

19. F. Feng, X. Y. Liu, L. F. Violeta and Y. B. Jun, Soft sets and soft rough sets, Inform. Sci. 181(2011)1125-1137.

20. Y. Y. Yao, Interval-set algebra for qualitative knowledge representation, Proc. of the 5th International Conference on Computing and Information, IEEE Computer Society Press, 1993, 370-375.

21. Y. Y. Yao and Q. Liu, A generalized decision logic in interval-set-valued information tables, Proc. of the 7th International Workshop on Rough Sets, Fuzzy Sets, Data Mining and Granular-Soft Computing, LNAI
1711, 1999, 285-293.

22. Y. Y. Yao and X. Li, Comparison of rough-set and Interval-set models for uncertain reasoning, Fundamenta Informaticae 27(1996)289-298.

23. Y. Y. Yao and S. K. M. Wong, Interval approaches for uncertain reasoning, Proc. of the 10th International Symposium on Methodologies of Intelligent Systems, LNAI 1325, 1997, 381-390.

24. R. E. Moore, Interval Analysis, Englewood Cliffs, New Jersey: Prentice-Hall, 1966.

25. Y. Y. Yao, Interval sets and interval-set algebras, Proc. of the 8th IEEE International Conference on Cognitive Informatics, 2009, 307-314.

26. R. A. Borzooei, M. Bakhshi and M. Mashinchi, Lattice structure on some fuzzy algebraic systems, Soft Computing 12(2008)739-749.

27. Y. Q. Wang and X. H. Zhang, Some implication operators on interval sets and rough sets, Proc. of the 8th IEEE International Conference on Cognitive Informatics, 2009, 328-332.

28. Y. Zou and Z. Xiao, Data analysis approaches of soft sets under incomplete information, Knowledge-Based Systems 21(2008)941-945. 